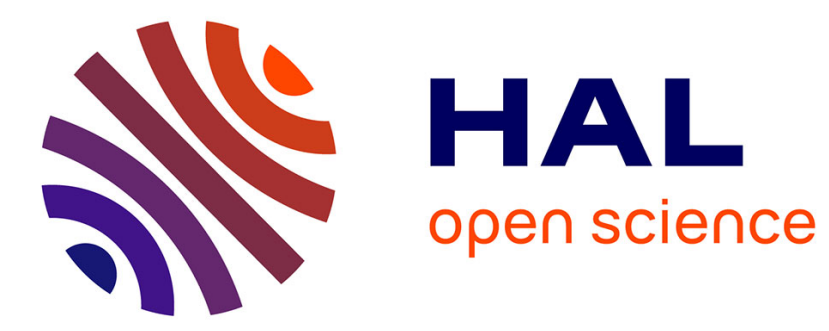

\title{
FingerMap: a new approach to predict soft material 3D objects printability
}

Christophe A. Marquette, Alix Lopez, Edwin-Joffrey Courtial

\section{To cite this version:}

Christophe A. Marquette, Alix Lopez, Edwin-Joffrey Courtial. FingerMap: a new approach to predict soft material 3D objects printability. Progress in Additive Manufacturing, 2020, 10.1007/s40964-02000143-5 . hal-03027622

\section{HAL Id: hal-03027622 \\ https://hal.science/hal-03027622}

Submitted on 11 Dec 2020

HAL is a multi-disciplinary open access archive for the deposit and dissemination of scientific research documents, whether they are published or not. The documents may come from teaching and research institutions in France or abroad, or from public or private research centers.
L'archive ouverte pluridisciplinaire HAL, est destinée au dépôt et à la diffusion de documents scientifiques de niveau recherche, publiés ou non, émanant des établissements d'enseignement et de recherche français ou étrangers, des laboratoires publics ou privés. 


\section{Progress in Additive Manufacturing FingerMap: A new approach to predict soft material 3D objects printability --Manuscript Draft--}

Manuscript Number:

Full Title:

Article Type:

Funding Information:

Abstract:

Corresponding Author:

Corresponding Author Secondary

Information:

Corresponding Author's Institution:

Corresponding Author's Secondary Institution:

First Author:

First Author Secondary Information:

Order of Authors:

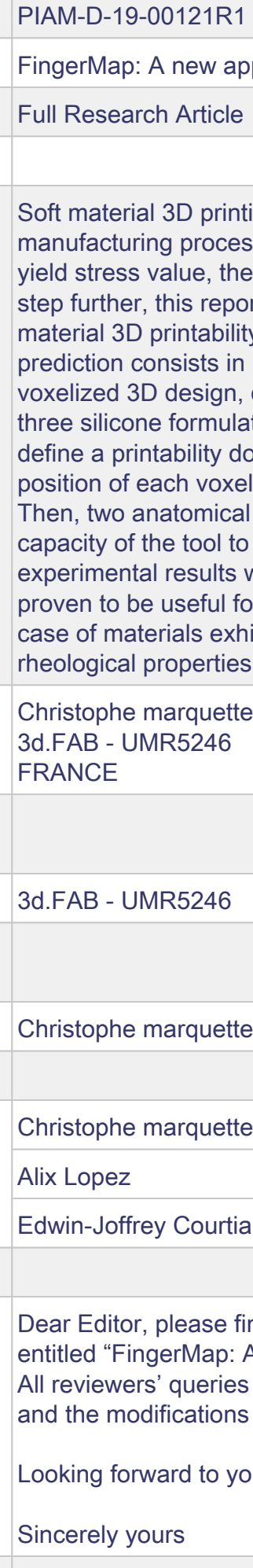

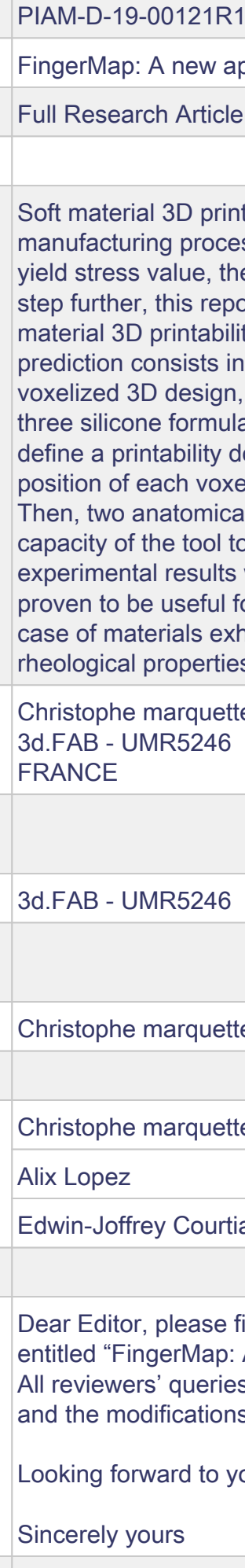

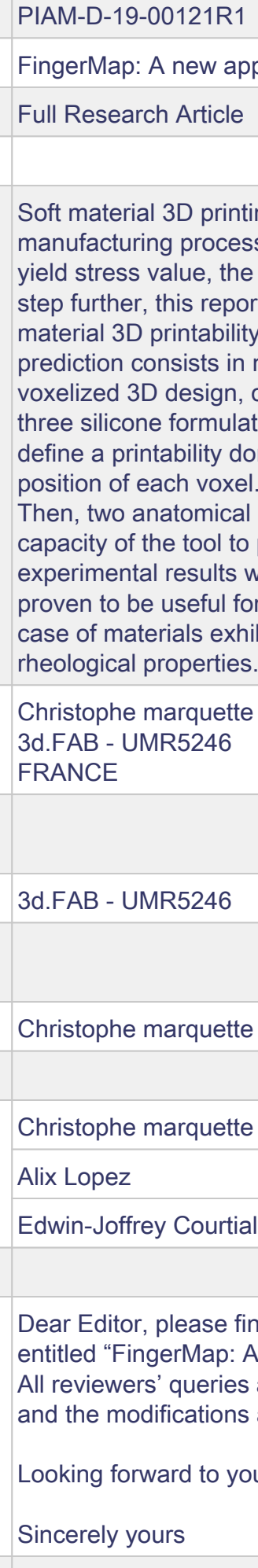

Order of Authors Secondary Information:

Author Comments:

Author Comments.

Response to Reviewers:

entitled "FingerMap: A new approach to predict soft material 3D objects printability".

All reviewers' queries and comments have been addressed, listed in a "Response" file and the modifications appear in red in the revised manuscript.

Looking forward to you decision.

Sincerely yours

All reviewers' queries and comments have been addressed, listed in a "Response" file and the modifications appear in red in the revised manuscript.

\section{Suggested Reviewers:}




\begin{abstract}
:
Soft material 3D printing through Liquid Deposition Modelling (LDM) is a challenging manufacturing process where yield stress control is mandatory. Indeed, the higher the yield stress value, the more complex the $3 \mathrm{D}$ printed structure can be. In a bid to go one step further, this report proposes a new approach enabling the prediction of soft material 3D printability as a function of the material's properties and shape. The prediction consists in numerical simulation to anticipate, in silico, the collapse of a voxelized 3D design, called FingerMap. To do so, a calibration of the program using three silicone formulations (with increasing yield stress value) was first performed to define a printability domain according to mass/surface ratio, overhang angle and the z-position of each voxel.

Then, two anatomical 3D models (ear and aortic valve) were used to demonstrate the capacity of the tool to predict printability. Good correlations between theoretical and experimental results were obtained. The proposed in silico simulation tool was then proven to be useful for LDM, even if some limitations were identified, particularly in the case of materials exhibiting complex rheological behaviours such as time-dependent rheological properties.
\end{abstract}




\section{Introduction}

In all fields of applications, soft materials provide unrivalled chemical or physical properties [1]. However, their use in manufacturing, and particularly additive manufacturing, processes can be difficult. This is the case of 3D printing of elastomers, which has the potential to create highly complex objects [2] with biomechanical properties easily tuned through formulations [3]. The complexity of using these elastomeric materials through additive manufacturing lies in understanding their rheological properties [4] throughout their state transition, from liquid to solid. Silicone is within this material category one of the most useful thanks to its potential application in multiple industrial sectors [5].

Silicone can be 3D printed through different processes [2] such as inkjet [6], Freeform Reversible Embedding of Suspended Hydrogels (FRESH) [7] or direct deposition [4, 8] also called Liquid Deposition Modelling (LDM). Of these three methods, one uses mandatory support material regardless of the printed shape (inkjet), one entails working with silicone complex rheological behaviours through the use of a suspending environment (FRESH), and one is highly dependent upon the material's rheological properties (LDM). The present study focuses on this latter method and on possible ways to enhance LDM success and productivity through the development of a numerical simulation tool. Indeed, LDM is usually facing printing success issues related to the fact that the printed material is not solidified right after printing and tends to collapse on its own weight.

Studies have already linked the shear yield stress (commonly called yield stress) to the material's printability when using the $\operatorname{LDM}$ technique $[9,10]:$ the higher the yield stress value, the more complex the 3D printed structures can be [4]. Previous studies have also demonstrated that the yield stress can be enhanced by creating, within the material, a weak secondary network with fast recovery [11]. Different yield stress agents were then investigated such as silica beads [12], glass fibres [13] or polyethylene glycol chains (PEG) [4]. The creation of a weak network by capillary forces was also investigated [14]. 
Even if the link between yield stress and object complexity has been clearly established experimentally [4], there is currently no numerical tool able to predict the printability of a 3D object according to the rheological properties of the printed material. The only available prediction tools are dedicated to thermoplastic materials and Fused Deposition Modelling (FDM). For instance, Finite Element Analysis (FEA) is used to calculate residual stress and distortion due to heat transfer during a 3D printing process, thus optimizing dimensional accuracy and preventing failure [15]. Distortion maps provided by FEA can be used as a mean to do reverse engineering and improve accuracy [16]. FEA can also be employed to predict behaviour under load conditions [17]. Structural analysis based purely on geometry can also be used to identify those areas that are the most likely to break [18], with the possibility of using an automatic geometric correction to reduce stress [19]. Massspring modelling [20] offers a first step for numerical simulation of soft material 3D structures. Less accurate than FEA, it is widely used for deformable bodies [21] [22], using a voxelbased approach and opening the path to multi-material simulation.

In the present study, a numerical simulation program was developed and validated as a pre-printing decision tool for soft material 3D printing. The program defines material printability, through shear yield stress value, and compares it with the voxelized 3D object (called FingerMap) to be printed. It then predicts the non-printability of the object by displaying a map of potential collapse areas. This tool is intended to allow users to anticipate in silico the material printability according to each 3D design, and not via the optimisation of printing parameters, thus reducing the number of tests required and accelerating the development of LDM technology using soft materials. 


\section{Material and methods}

2.1. Preparing and curing silicone elastomer formulations

Silbione ${ }^{\circledR}$ LSR 4350 from Elkem Silicones is a bi-component formulation, with parts $A$ and $B$ to be mixed in equal parts $(1: 1)$ at room temperature. It is composed of different polydimethylsiloxane (PDMS) chains mixed with fumed silica particles. Part B contains a high concentration of inhibitor agent intended to guarantee a high pot life $(>24 \mathrm{~h})$ after mixing. This is crucial in order to ensure non-evolving rheological properties throughout the 3D printing process. Three formulations of Silbione® LSR 4350 were used. The first one was the crude material, the second was the material mixed with PEG (Mw=400 $\mathrm{g} / \mathrm{mol}$ ) (Sigma-Aldrich, USA) and the third formulation was material mixed with BLUESIL® SP 3300 (Elkem Silicones, France), a non-hydrolysable silicone polyether copolymer (Supplementary Information 1). PEG and SP 3300 were used to custom the static yield stress value of crude material. These yield stress agents display an optimum content in silica/PDMS suspensions, so mass fractions of yield stress agents were optimised prior to this study [23, 24]. Their addition in the LSR was performed in part A with a mass fraction of $2 \%$ for the PEG and $2.7 \%$ for the SP. Then, parts $A$ and $B$ were mixed $1: 1(w / w)$ at room temperature. After mixing, samples were loaded in $10 \mathrm{cc}$ cartridges (Nordson® EFD, USA) and degassed through centrifugation at 5000 rpm for 5 minutes (ProcessMate ${ }^{\circledR} 5000$, Nordson EFD, USA).

After printing, 3D structures were thermally cured in a 3-step protocol: room temperature for $72 \mathrm{~h}, 60^{\circ} \mathrm{C}$ for $4 \mathrm{~h}$ and finally $200^{\circ} \mathrm{C}$ for $2 \mathrm{~h}$.

2.2. Rheological characterisation of silicone elastomer formulations

Rheology measurements were conducted using a stress-controlled Discovery HR-2 rheometer (TA Instruments, USA) with a plate-plate geometry of $40 \mathrm{~mm}$ in diameter. Characterization was performed just after mixing and degassing. 
In the present study, the normal yield stress [25] and the yield stress tensor [26] were measured $\tau_{y}^{s}$ was close to normal yield stress. $\tau_{y}^{s}$ values were obtained using $10^{-4}-1 \mathrm{~s}^{-1}$ and $1-10^{-4} \mathrm{~s}^{-1}$ shear rate sweeps, measuring five points per decade with an averaging time of 30 seconds. Only the shear rate sweep from $1-10^{-4} \mathrm{~s}^{-1}$ was used to plot curves in order to predict thixotropic behaviour and obtain reproducible results [27]. The experiment was performed at $25^{\circ} \mathrm{C}$. The $\tau_{y}^{s}$ was obtained by extrapolation of shear stress at zero shear rate using the well-known Herschel-Bulkley model (Equation 1) [28] available with TRIOS software (TA instruments, USA) in analysis tools.

$\tau=\tau_{y}^{s}+k \dot{\gamma}^{n}$

Equation 1: Constitutive equation of the Herschel-Bulkley model. $\tau$ is the shear stress, $\tau_{y}^{s}$ the shear static shear stress, $\dot{\gamma}$ the shear rate, $k$ the consistency index and $n$ the flow index [28]

\section{3. $3 D$ designs}

Four 3D designs were used during this study (Fig. 1). The first two designs were calibration objects, namely the tube (Fig. 1.A, $10 \mathrm{~mm}$ internal diameter, $20 \mathrm{~mm}$ external diameter) and the test angle which is a structure with increasing overhang (Fig. 1.B). These were printed to correlate printability and the measured $\tau_{y}^{s}$ of each silicone elastomer formulation. Tubes were printed of varying heights in order to determine the highest mass/surface ratio each formulation could bear before losing dimensional accuracy. Each tube was weighted in order to experimentally determine the mass causing the distortion. The test angle, a comb-like structure with increasing overhang (from 20 to $75^{\circ}$ with $5^{\circ}$ step), was printed to define the critical overhang for which the material printability was not guaranteed. Dimensional accuracy was monitored by callipers-based measurements representative of each geometry.

The other two designs were evaluation objects, namely an ear (Fig. 1.C) and an aortic valve (Fig. 1.D). These ones were used to validate the result given by our predictive tool. The aortic valve presents thin overhangs with relatively low mass/surface ratio (12.15 
$\mathrm{mg} / \mathrm{mm}^{2}$ ) whereas the ear is more complex, with a strong overhang of $60^{\circ}$ ( $0^{\circ}$ being vertical)

A.

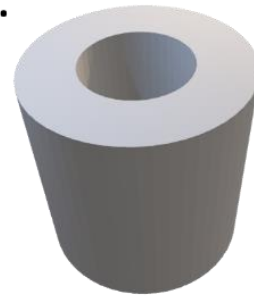

C.

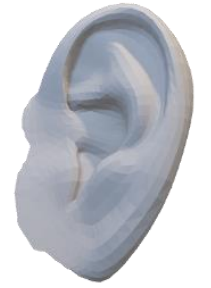

B.

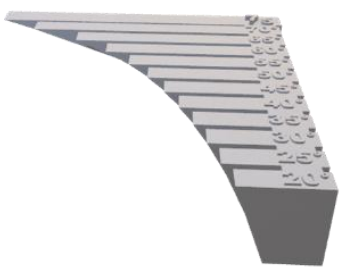

D.

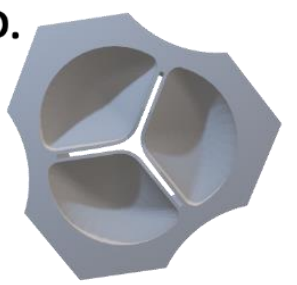

Fig. 1. The different files used for the study. A. Tube STL used for determination of the maximal mass/surface ratio. B. Test angle (from $20^{\circ}$ to $75^{\circ}$ ) STL used for determination of the maximal angle overhang. C. Human ear STL used for comparison with FingerMap. D. Aortic valve STL used for comparison with FingerMap.

2.4. 3D printing of silicone formulations: Printer, dispenser and slicer

To print the three formulations (i.e., LSR, LSR+PEG and LSR+SP), a COSMED 333 Cartesian 3D printer (Tobeca $\AA$, France) was used. This printer possesses a movement precision of $10 \mu \mathrm{m}$. All formulations were loaded in 10cc cartridges equipped with a standard piston and a $200 \mu \mathrm{m}$ frustoconical nozzle (Nordson EFD, USA). The dispensing flow rate was controlled by an Ultimus $V$ pneumatic equipment (Nordson EFD, USA). The dispensing pressure was adapted for each formulation in order to match the printing speed. An additional tool was required, the $\mathrm{HPx}^{\mathrm{TM}}$ dispensing tool (Nordson EFD, USA), for the LSR+PEG and LSR+SP formulations. The printing was controlled with Repetier Host software (Repetier, V2.0.1, Germany). The slicing of 3D objects was performed using Slic3r software (Slic3r, V1.3.0) using appropriated printing parameters (Supplementary Information 2). Ear and aortic valve models have also been printed using an Objet Pro inkjet printer (Stratasys, USA) with VeroClear resin (Stratasys, USA) in order to have reference geometries for comparison.

2.5. The predictive tool: FingerMap versus Printability volume 
A custom MATLAB (MathWorks R2018B, USA) code was used in this study. The code uses an STL file as an input and indicates troublesome areas to print on the basis of the measured $\tau_{y}^{s}$ and the known formulation density. To briefly resume, the code first voxelizes the STL file (each point of the object volume being represented by a voxel), then treats each voxel to determine its carried mass, detects its overhanging status and determines its overhanging angle value. From this voxelization, the Fingermap of the 3D object can be built. The prediction level is given when the FingerMap is compared to the printability volume as a function of material rheological properties. The voxel displays as red if the $\tau_{y}^{s}$ is not high enough to withstand the shape complexity. Otherwise, the voxel displays as green. 


\section{Theory}

This section describes the overall mechanism of our predictive tool. A general description is given through an explanation of how the geometric analysis is performed.

\subsection{FingerMap and printability}

To work, the software requires three kinds of input. First, it needs material inputs: the mass density and $\tau_{y}^{s}$ of the printed material. Mass density is used to calculate the mass/surface ratio while the $\tau_{y}^{s}$ is used to determine the printability of the material. Next, it requires a geometrical input, an STL file, in order to analyse the geometry and determine if it is compatible with the material's printability. Finally, the desired resolution is required as an analytical input. It defines the precision (resolution value) at which the analysis will be performed. This resolution must be equivalent to the printing parameters (layers' height and width) to be compared with experimental data.

Once launched, the software begins by creating a 3D grid around the STL to determine if each point is inside or outside the volume. All points inside the volume are represented by a voxel, whose size is defined by the resolution value, resulting in a voxelized object. Each voxel is then analysed to determine its load and its mass/surface ratio, its overhang and its position along the $z$-axis as the position indicator.

The software provides three outputs. The first output is the FingerMap object. Similar to the human fingerprint, a FingerMap is unique and characteristic of a distinct geometry. A FingerMap consists of a 3D scatter graph where each voxel is represented by a point with coordinates corresponding to its mass/surface ratio, its overhang and its $z$ coordinate. The second output is the material printability map which represents, in the same coordinate (mass/surface ratio, overhang, z-axis), a volume that contains all the printable points according to the material $\tau_{y}^{s}$. The third and last output is a visualisation of voxels that can 
(green colour) or cannot (red colour) be perfectly printed, namely all the dots from the FingerMap that are respectively inside or outside the printability volume.

3.2. Calculation of mass/surface ratio

As a first approach, we made a simplified theory to evaluate the mass/surface ratio of each voxel by assuming that this voxel only supports the weight of its upper neighbours. In this method, the software finds the next highest voxel that does not possess an upper neighbour and counts how many voxels separate them. Using the mass density, the mass of a voxel can be easily determined using the resolution value to calculate the volume, thus allowing us to calculate the mass supported by a voxel by multiplying it by the number of voxels above it. From the resolution value, we can also determine the surface and lastly the mass/surface ratio.

\subsection{Identification and calculation of the overhang angle}

To reduce computing time, unsupported voxels were detected and subjected to an overhang analysis. To determine the overhanging angle, the MATLAB functions incenter() and faceNormal() were used in order to extract the centre coordinates and the normal of each triangle from the STL (as illustrated in Fig. 2). The distance between the voxel and each centre was then calculated in order to find the nearest facet and assign its normal to the voxel. The angle with the vertical was then calculated and used to plot the FingerMap. The feasibility of our overhang calculation strategy was verified by using the test angle STL file and comparing the overhang results given by its FingerMap and the known values for the angles from the STL (Supplementary 3). 


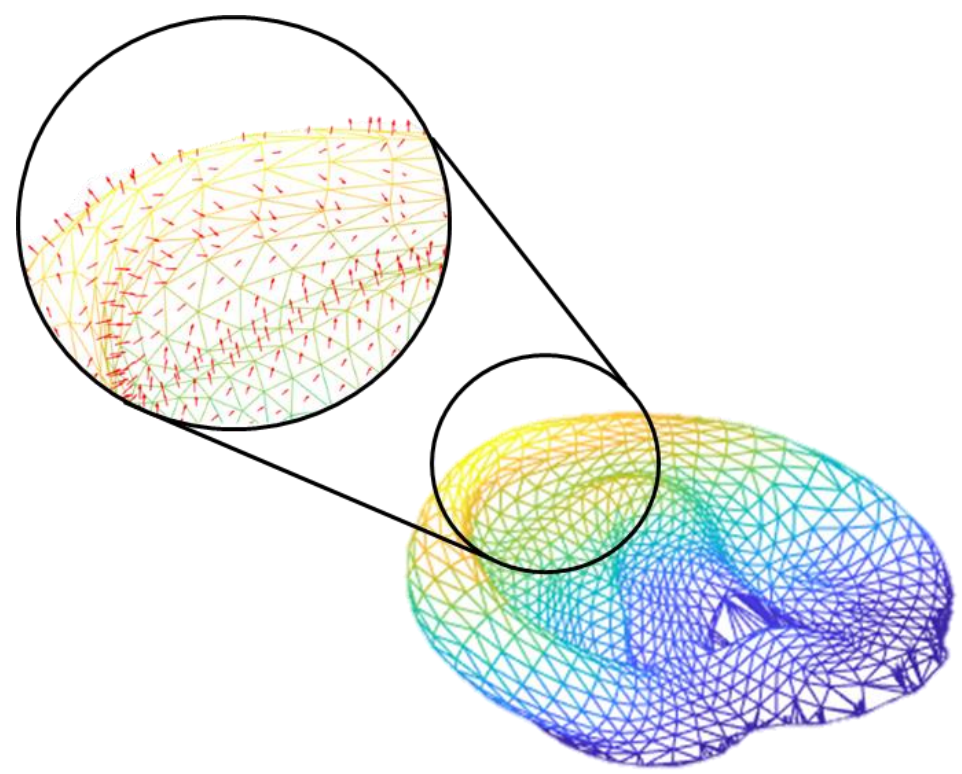

Fig. 2. Overhang calculation concept, from centre and normal identification of each triangle. Case study through STL file of human ear. 


\section{Results}

As described in the Theory section, the developed program experimentally determines the rheological properties of different materials together with the limit printable geometric features (mass/surface ratio and overhang). Once this information has been gathered, 3D prints can be performed and displayed alongside the simulations yielded by the software.

\subsection{Rheological properties of silicone formulations}

The yield stress character of each formulation is illustrated in Fig. 3, with curves exhibiting the characteristic threshold value required for the material to flow. It displays, for each of them, the experimental points as well as the Herschel-Bulkley model (Equation 1) with its coefficient of determination and the extracted $\tau_{y}^{s}$ value. The calculated $\tau_{y}^{s}$ of LSR, LSR+PEG and LSR+SP formulations are 168, 600 and $1397 \mathrm{~Pa}$, respectively. According to a previous study [4], the LSR+SP formulation would seem to be the best adapted for 3D printing. Of important note however within the context of this study is that the three tested materials exhibit very different static yield stresses and are therefore good candidates to challenge the simulation software. 
Fig. 3. Static Shear Yield Stress $\left(\tau_{y}^{s}\right)$ determination of silicone formulations through the Herschel-Bulkley model (dotted blue line describe the asymptote). $\tau_{y}^{s}$ values are given with a correlation coefficient $\left(R^{2}\right)$ calculated by Trios software (TA Instruments).

4.2. Determination of the maximum printable features from calibration objects.

Table 1 summarises the results in terms of maximum printable features. From tube printing experiments, the maximum mass/surface ratio was considered attained as soon as a height difference of more than a layer thickness $(200 \mu \mathrm{m}$ in our case) between the printed tube and the STL file was observed. From test angle printing experiments, the maximum overhang angle was considered attained when a loss of parallelism between the top and bottom surface of the object was detected, proof of a collapsed overhang. All experimental results are available in supplementary information 4.

Yield stress effect on printability is here once again clearly evidenced since enhancing this rheological property through formulation allowed the LSR, with the addition of BLUESIL® SP 3300 , to increase its maximum mass/surface ratio from 3.99 to $44.05 \mathrm{mg} / \mathrm{mm}^{2}$ and its overhang print capability from $0^{\circ}$ to $55^{\circ}$. 
Table 1. Shear Static Yield Stress and maximum printable features of silicone formulations. Three tests were performed for each data with a standard deviation less than $1 \%$.

\begin{tabular}{|c|c|c|c|}
\hline Formulation & $\tau_{y}^{s}(\mathrm{~Pa})$ & Maximum mass/surface ratio $\left(\mathrm{mg} / \mathrm{mm}^{2}\right)$ & Maximum overhang $\left(^{\circ}\right)$ \\
\hline LSR & 168 & 3.99 & 0 \\
\hline LSR + PEG & 600 & 16.28 & 45 \\
\hline$L S R+S P$ & 1397 & 44.05 & 55 \\
\hline
\end{tabular}

4.3. FingerMap versus Printability volume

From our algorithm, both anatomical geometries STL files (Fig. 4.A \& Fig. 5.A) were voxelized to establish their FingerMap with a resolution of $0.2 \mathrm{~mm}$ and a volumetric mass of $1.125 \mathrm{~g} / \mathrm{cm}^{-3}$. The resulting voxelizations are illustrated in Fig. 4.B \& Fig. 5.B. (Software outputs), alongside 3D printing results, and are displayed in Fig. 5.D for the aortic valve and in Fig. 4.D for the ear. As stated in section 3.1, the FingerMap (blue scattered points) does not change for a distinct object given the fact that it only relies on geometry and the material's mass density, which in our case does not vary with the addition of yield stress agent.

The printability volume, identify by the green plane threshold, was defined using the characteristic values defined in Table 1 . Blue dots outside this printability volume represent voxels that have geometric requirements incompatible with the material's $\tau_{y}^{s}$, in other words, areas that will not be printed properly. These voxels are displayed in red while the nonproblematic ones are green. Thus, we verified that the lower the material's $\tau_{y}^{s}$ (i.e., the smaller the printability volume), the higher the number of not-printable voxels (greater proportion of red voxels). 


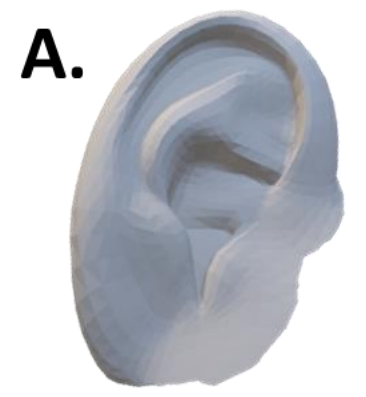

B.

D.
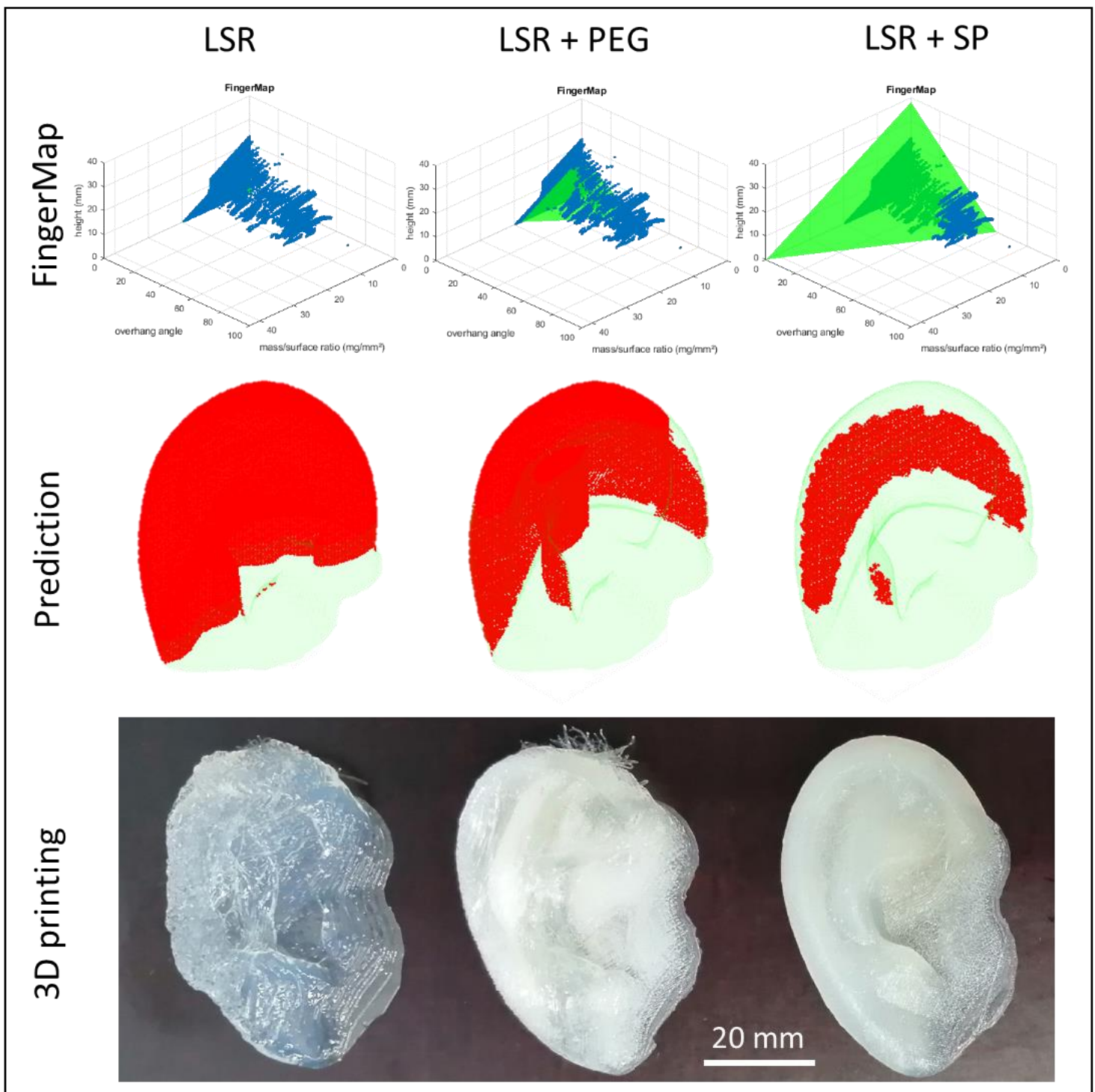

Fig. 4. Comparison between the experimental data (3D printing) and the results given by FingerMap for the human ear. A. Human ear STL file used. B. Voxelized volume. The resolution value used was $0.2 \mathrm{~mm}$. C. Human ear control print using ObjetPro. D. FingerMap vs Material printability volume. The simulation and the experimental 3D print obtained for each formulation. The green volume represents the printability of the material. Each blue dot represents a voxel. In the prediction, red voxels represent not-printable area while green voxels represent printable area. 

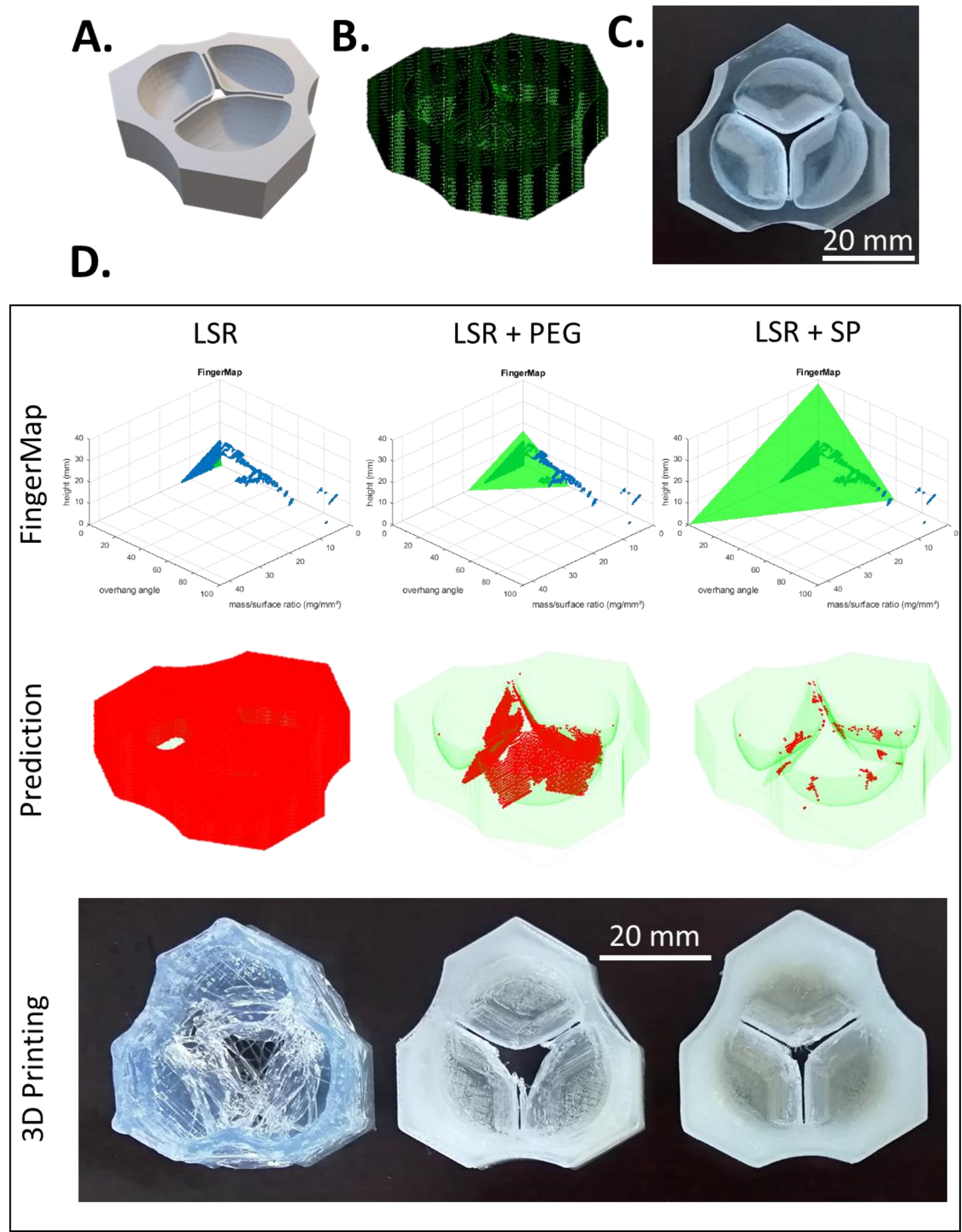

Fig. 5. Comparison between the experimental data (3D printing) and the results given by FingerMap for the aortic valve. A. Aortic valve STL file used. B. Voxelized volume. The resolution value used was $0.2 \mathrm{~mm}$. C. Aortic valve control print using ObjetPro. D. FingerMaps vs Material printability volume. The simulation and the experimental 3D print obtained for each formulation. The green volume represents the printability of the material. Each blue dot represents a voxel. In the prediction, red voxels represent not-printable area while green voxels represent printable area. 
4.4. Dimensional analysis of the evaluation 3D objects printing

Ear 3D printing analysis are illustrated in Fig. 6.A. Both 3D printed ears produced using LSR and LSR+PEG totally collapsed (respectively 11.5 and $10.9 \mathrm{~mm}$ high instead of $22 \mathrm{~mm}$ ), which is consistent with results displayed in Fig. 4 since the STL is characterized by an overhanging angle of $60\left(>45^{\circ}\right)$. However, the failure occurred differently using the two formulations. Indeed, the LSR+PEG ear was printed successfully and then collapsed before curing, whereas the LSR ear collapsed during printing. As a result, LSR+PEG shows a better shape accuracy in the failed area (as seen in Fig. 4.D). The LSR+SP formulation also collapsed since the overhang still exceeds the material's $55^{\circ}$ limit, but it displays the better geometric accuracy and is the closest to the control shape. These results are in agreement with the Fig. 4.D FingerMap results where a minority of voxel are identified as non-printable for the LSR+SP formulation.

For the aortic valve 3D printing analysis, a measurement was performed on the object height in order to verify the collapse of the material. Fig. 6.B depicts the side views of the obtained objects. As can be seen, the difference of print heights of 0.7 and $0.5 \mathrm{~mm}$ were obtained for the LSR and LSR+PEG formulations, respectively. These values are significantly different from the expected $11.3 \mathrm{~mm}$. By contrast, the aortic valve printed using LSR+SP did have the expected $11.3 \mathrm{~mm}$ height. The top view of the printed objects (Fig. 5.D) gives more information since it shows the collapse of the inner structure. Once again, LSR+SP gives the best results and does not display shape distortion whereas the other two prints do. The LSR print exhibits the worst geometrical accuracy with valves not being formed at all and the outer structure thinner than the control print ( $3 \mathrm{~mm}$ instead of $4.9 \mathrm{~mm})$. The object obtained using LSR+PEG presents an intermediate accuracy with inner structures partially built. 


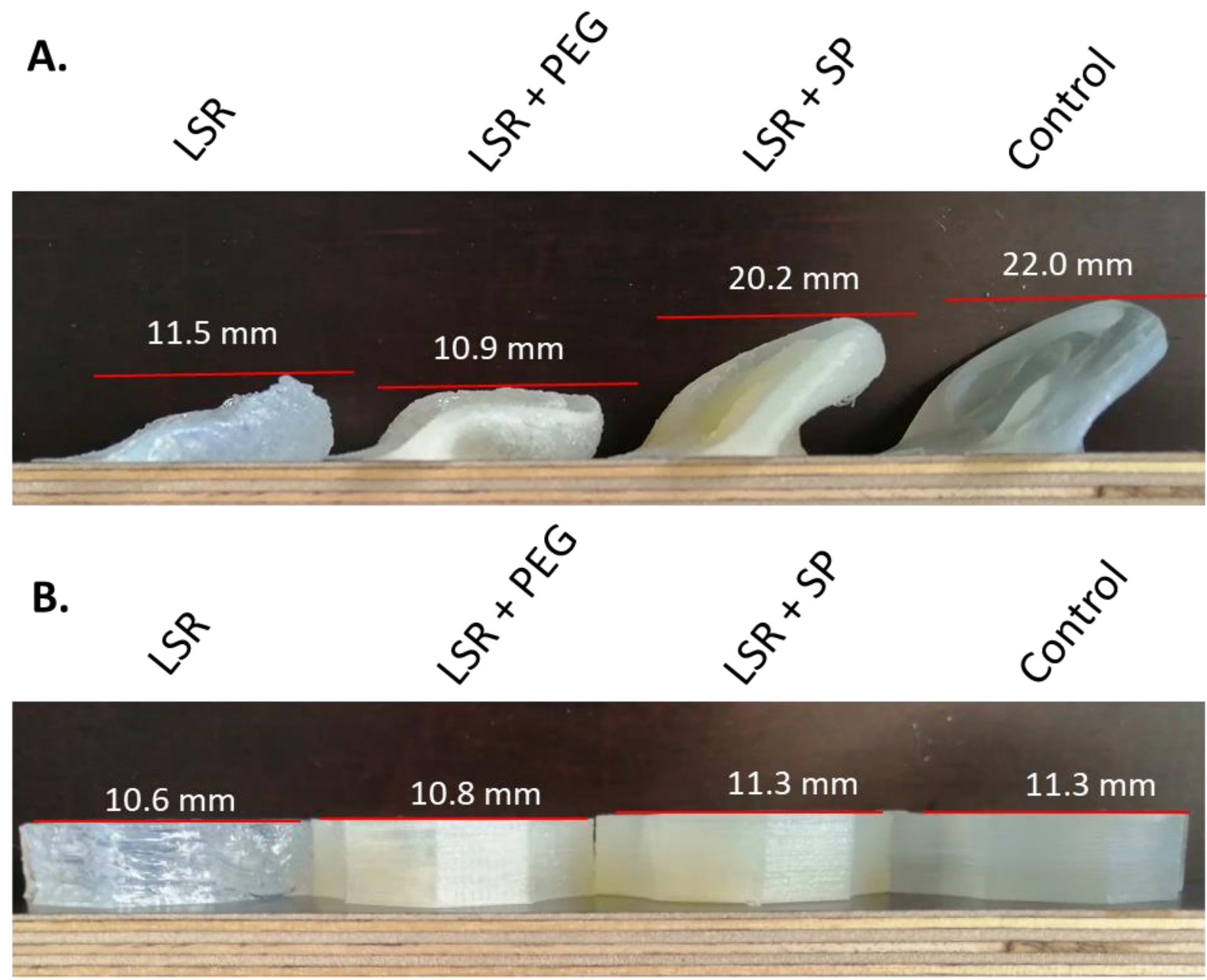

Fig. 6. 3D printing dimensional analysis after complete curing. A. 3D printed ears obtained using the three different formulations next to the control print. Numbers represent the height of the overhanging $3 D$ structure. $B .3 D$ printed aortic valves obtained using the three different formulations next to the control print. Numbers represent the height of the 3D structure. Three 3D printed experiments were performed for each object with a standard deviation less than $2 \%$.

\section{Discussion}

From a general point of view, LSR, with the lowest $\tau_{y}^{s}(168 \mathrm{~Pa})$, was not able to print high structure (maximal mass/surface ratio of $3.99 \mathrm{mg} / \mathrm{mm}^{2}$ ) or overhang. Low height areas, such as the bottom of the valves or the ear base were printed successfully as predicted by the simulation software since the geometric requirements matched the printability of the material. For the LSR+PEG formulation, the collapse of valves and ear overhangs was predicted by the software since they had an angle exceeding the maximum printable overhang $\left(>45^{\circ}\right)$. Since LSR+PEG had a higher mass/surface ratio limit than LSR (16.23 $\mathrm{mg} / \mathrm{mm}^{2}>3.99 \mathrm{mg} / \mathrm{mm}^{2}$, respectively), we considered that the outer structure of the aortic 
valve was printed properly, with the software predicting more accurately in the ear centre. Lastly, the LSR+SP formulation, with the highest yield stress $(1397 \mathrm{~Pa})$, had the best printability (maximum mass/surface ratio of $44.05 \mathrm{mg} / \mathrm{mm}^{2}$ and overhang of $55^{\circ}$ ); thus, the algorithm's predictions were almost perfect for the aortic valve and the collapse of the steepest part of the ear overhang.

In order to quantify the printing accuracy, strategic calliper-based measurements were performed on characteristic dimensions (supplementary information 5) and the mean relative deviation between the theoretical geometries, given by STL files, and the printed objects were extracted (Table 2). Predicted accuracy was also defined as the percentage of non-printable voxels identified using FingerMap (supplementary information 6). A first observation is that both accuracy values, predicted and measured, increase with the static shear yield stress value.

However, some discrepancies appear between predicted (non-pintable voxel) and measured values (mean relative deviation), particularly in the case of LSR and LSR+PEG. Indeed, when trying to print the ear file, identical mean relative deviation values $(\approx 27 \%)$ were obtained although the LSR+PEG material-based print exhibits greater accuracy (nonprintable voxels of $L S R+P E G=27 \%$ ) compared to $L S R$ (non-printable voxels $=84 \%$ ). Such a result demonstrates the limitations of our predictive tool. Indeed, when a voxel area is identified as non-printable, the impacted neighbours' voxels are not predicted. If a voxel collapses, all upper voxels should also collapse and then be identified as such in the FingerMap (red voxel), which was not the case. For example, in Fig. 4.D, the ear overhang (for the LSR+SP formulation) is red but its upper part is still green. In such case, our prediction approach can be defined as a local failure (voxel by voxel) identification tool. Furthermore, the non-printable label is unable to evaluate the level of collapse and the origin of failure, since our approach was only phenomenological. Indeed, when the collapse begins, the mass/surface ratio and overhang of local material area changes. 
It is important to note that our approach only works for material displaying stable rheological properties over the printing time. Any material evidencing rheological changes during the printing duration cannot be computed correctly since our predictive tool requires a constant $\tau_{y}^{s}$ value.

To push the limits of this new approach, the assumption of steady state would need to be lifted. In this way, it could be interesting to develop a new strategy using high precision 3D imaging tools in additive manufacturing process to compare printed and native numerical object. This "live" strategy would be able to evaluate the impact of non-printable and printable voxels during printing of calibration object. The addition of voxel's evolution in time appears; i.e., temporality, will then allow us to include, in the prediction software, the more complex rheological properties such as elongational or thixotropic behaviours and their evolution. The use of STL format as input would also appear to be a limitation, since it reflects the static state of an object, compared to g.code format which includes time-dependent information.

Table 2. Accuracy, defined as the percentage of predicted non-printable voxels and as the mean relative deviation, which is the sum of relative errors (supplementary information 5), between printed and theoretical dimension (STL file).

\begin{tabular}{|l|c|c|c|c|c|}
\hline & & \multicolumn{2}{|c|}{ Ear } & \multicolumn{2}{c|}{ Aortic valve } \\
\hline & $\boldsymbol{\tau}_{\boldsymbol{y}} \mathbf{( P a )}$ & $\begin{array}{c}\text { Non-Printable } \\
\text { voxel (\%) }\end{array}$ & $\begin{array}{c}\text { Mean relative } \\
\text { error (\%) }\end{array}$ & $\begin{array}{c}\text { Non-Printable } \\
\text { voxel (\%) }\end{array}$ & $\begin{array}{c}\text { Mean relative } \\
\text { error (\%) }\end{array}$ \\
\hline LSR & 168 & 83.97 & 26.96 & 88.16 & 14.88 \\
\hline LSR + PEG & 600 & 26.53 & 27.39 & 1.77 & 4.24 \\
\hline LSR + SP & 1397 & 2.92 & 4.39 & 0.10 & 1.12 \\
\hline
\end{tabular}

Nevertheless, as a first-generation prediction tool, the FingerMap approach was shown to be an effective instrument to anticipate the printability of soft material as a function of the 3D designs. FingerMap seems to us to be the only original concept, with a strong growth development, able to facilitate the accessibility of soft material 3D printing and accelerate its industrial development. 


\section{Conclusion}

In the present work, we developed a numerical tool to predict the failure of LDM 3D printing. Three silicone formulations with different static shear yield stress values were used to demonstrate the program's capabilities.

- This predictive tool voxelizes an STL file, characterises each voxel in terms of mass/surface ratio, overhang angle and position regarding the z-axis and then formulates a FingerMap view of the object. By overlapping this FingerMap analysis to the printability volume define by the $\tau_{y}^{s}$ of the material, the tool is able to predict which voxel are not printable.

- Two case studies of complex anatomical geometries (ear and aortic valve) were performed in order to compare the prediction results and the experimental data.

- The obtained results were more than satisfactory and demonstrate the merits of our numerical approach.

In future works, we will investigate other rheological properties based on temporality such as thixotropy or elongational viscosity, to allow users to anticipate the LDM printability of 3D objects using more complex materials. 


\section{References}

1. MarketsAndMarkets.com (2016) Elastomers Market by Type (Thermoset (Natural Rubber, Synthetic Rubber (SBR, IIR, PBR, NBR, ACM, EPM)), and Thermoplastic (PEBA, SBC, TPO, TPU, TPV)), Application (Automotive, Consumer Goods, Medical, and Industrial) - Global Forecast to 2021

2. Liravi F, Toyserkani E (2018) Additive manufacturing of silicone structures: A review and prospective. Addit Manuf 24:232-242. https://doi.org/10.1016/J.ADDMA.2018.10.002

3. Corbett TJ, Doyle BJ, Callanan A, et al (2010) Engineering Silicone Rubbers for In Vitro Studies: Creating AAA Models and ILT Analogues With Physiological Properties. J Biomech Eng 132:011008. https://doi.org/10.1115/1.4000156

4. Courtial E-J, Perrinet C, Colly A, et al (2019) Silicone rheological behavior modification for 3D printing: Evaluation of yield stress impact on printed object properties. Addit Manuf 28:5057. https://doi.org/10.1016/J.ADDMA.2019.04.006

5. Corden C, Tyrer D, Menadue H, et al (2016) Socio-economic evaluation of the global silicones industry

6. McCoul D, Rosset S, Schlatter S, Shea H (2017) Inkjet 3D printing of UV and thermal cure silicone elastomers for dielectric elastomer actuators. Smart Mater Struct 26:125022. https://doi.org/10.1088/1361-665X/aa9695

7. Hinton TJ, Hudson A, Pusch K, et al (2016) 3D Printing PDMS Elastomer in a Hydrophilic Support Bath via Freeform Reversible Embedding. ACS Biomater Sci Eng 2:1781-1786. https://doi.org/10.1021/acsbiomaterials.6b00170

8. Plott J, Shih A (2017) The extrusion-based additive manufacturing of moisture-cured silicone elastomer with minimal void for pneumatic actuators. Addit Manuf 17:1-14. https://doi.org/10.1016/j.addma.2017.06.009

9. Mouser VHM, Melchels FPW, Visser J, et al (2016) Yield stress determines bioprintability of hydrogels based on gelatin-methacryloyl and gellan gum for cartilage bioprinting. Biofabrication 8:1-24. https://doi.org/10.1088/1758-5090/8/3/035003

10. Hiller A, Borchers K, Tovar GEM, Southan A (2017) Impact of intermediate UV curing and yield stress of 3D printed poly(ethylene glycol) diacrylate hydrogels on interlayer connectivity and maximum build height. Addit Manuf 18:136-144. https://doi.org/10.1016/j.addma.2017.10.008

11. Li L, Lin Q, Tang M, et al (2019) Advanced Polymer Designs for Direct-Ink-Write 3D Printing. Chem - A Eur J chem.201900975. https://doi.org/10.1002/chem.201900975

12. Aranguren MI, Mora E, Macosko CW, Saam J (1994) Rheological and Mechanical Properties of Filled Rubber: Silica-Silicone. Rubber Chem Technol 67:820-833.

https://doi.org/10.5254/1.353871

13. Kotsilkova R, Gleisslex W (1990) A Study of Transient and Steady-State Shear and Normal Stresses in Glass Fiber Suspensions. In: Third European Rheology Conference and Golden Jubilee Meeting of the British Society of Rheology. Springer Netherlands, Dordrecht, pp 280282

14. Roh S, Parekh DP, Bharti B, et al (2017) 3D Printing by Multiphase Silicone/Water Capillary Inks. Adv Mater 29:1701554. https://doi.org/10.1002/adma.201701554 
15. Mukherjee T, Zhang W, DebRoy T (2017) An improved prediction of residual stresses and distortion in additive manufacturing. Comput Mater Sci 126:360-372.

https://doi.org/10.1016/J.COMMATSCI.2016.10.003

16. Yaghi A, Ayvar-Soberanis S, Moturu S, et al (2019) Design against distortion for additive manufacturing. Addit Manuf 27:224-235. https://doi.org/10.1016/j.addma.2019.03.010

17. Kadkhodapour J, Montazerian H, Raeisi S (2014) Investigating internal architecture effect in plastic deformation and failure for TPMS-based scaffolds using simulation methods and experimental procedure. Mater Sci Eng C 43:587-597.

https://doi.org/10.1016/j.msec.2014.07.047

18. Zhou Q, Panetta J, Zorin D (2013) Worst-case Structural Analysis. ACM Trans Graph 32:137:1-137:12. https://doi.org/10.1145/2461912.2461967

19. Stava O, Vanek J, Benes B, et al (2012) Stress relief: Improving structural strength of 3D printable objects. ACM Trans Graph 31:45-46. https://doi.org/10.1145/2185520.2185544

20. Golec K (2018) Hybrid 3D Mass Spring System for Soft Tissue Simulation. Universite Lyon 1

21. Sossou G, Demoly F, Belkebir H, et al (2019) Design for 4D printing: A voxel-based modeling and simulation of smart materials. Mater Des 175:107798.

https://doi.org/10.1016/j.matdes.2019.107798

22. Hiller J, Lipson H (2014) Dynamic Simulation of Soft Multimaterial 3D-Printed Objects. Soft Robot 1:88-101. https://doi.org/10.1089/soro.2013.0010

23. Paquien J-N (2003) Etude des propriétés rhéologiques et de l'état de dispersion de suspensions PDMS/Silice: rheological properties and dispersion of PDMS/silica suspensions. Lyon, INSA

24. Paquien JN, Galy J, Gérard JF, Pouchelon A (2005) Rheological studies of fumed silicapolydimethylsiloxane suspensions. Colloids Surfaces A Physicochem Eng Asp 260:165-172. https://doi.org/10.1016/j.colsurfa.2005.03.003

25. de Cagny H, Fazilati M, Habibi M, et al (2019) The yield normal stress. J Rheol (N Y N Y) 63:285-290. https://doi.org/10.1122/1.5063796

26. Thompson RL, Sica LUR, de Souza Mendes PR (2018) The yield stress tensor. J Nonnewton Fluid Mech 261:211-219. https://doi.org/10.1016/J.JNNFM.2018.09.003

27. Ovarlez G (2011) Caractérisation rhéologique des fluides à seuil. Rhéologie 20:28-43

28. Herschel WH, Bulkley R (1926) Konsistenzmessungen von Gummi-Benzollösungen. KolloidZeitschrift 39:291-300. https://doi.org/10.1007/BF01432034 
Click here to access/download Supplementary Material

Lopez and al. - Supplementary information.docx 
COMMENTS TO THE AUTHOR:

Reviewer \#2: The authors developed a simulation program to predict the printability of soft material in Liquid Deposition Modelling (LDM) 3D printing. This numerical simulation program provided 3D printability information of three silico-based materials before 3D printing, in addition to other strategies, such as adjusting 3D printing parameters during and/or after real 3D printing. The printability trend of these three 3D printed ear and aortic valve matches the simulation results. Results from this paper are helpful for other researchers in this field to promote research in LDM using soft materials. Overall, this manuscript is well written and fits the scope of this journal, so I suggest publishing it with minor revisions if the concerns and questions below are addressed.

1. In the Materials and Methods section, can the authors explain why PEG ( $M W=400 \mathrm{~g} / \mathrm{mol}$ ), this specific material, was chosen to mix with silicone? PEG is also a commonly used soft material. How about testing the printability of pure PEG as a fourth material for comparison?

PEG 400 was used as yield stress agent to custom the static yield stress value of crude silicone. PEG 400 was already used in previous work (Courtial E-J, Perrinet C, Colly A, et al (2019) Silicone rheological behavior modification for 3D printing: Evaluation of yield stress impact on printed object properties. Addit Manuf 28:50-57. https://doi.org/10.1016/J.ADDMA.2019.04.006). To make this point clear, we have added the sentence: "PEG and SP 3300 were used to custom the static yield stress value of crude material".

Yield stress agents (PEG 400 and SP 3300) can't be printed alone due to their physical state which is liquid without yield stress behavior. Other PEG formulations used in $3 \mathrm{~d}$ printing are often PEGDA for UV curing and can't be compare here.

2. In Fig. 3., please correct "Buckley" into "Bulkley" and please correct all similar issues throughout this manuscript.

Many thanks. Our apologies for this mistake. We have made correction on Fig.3 and Eq.1.

3. For the data displayed in table 1, how many tests were performed for each data, will it be possible to display data as average \pm standard error?

"Three tests were performed for each data with a standard deviation less than 1\%". This description has been added in Table 1.

4. For Fig 4. And Fig 5, how many 3D printed experiments were performed to test the printability? Will it be possible to add more tests for each model (e.g, the ear) to verify the prediction ability of this simulation method and make the conclusion sounder?

Due to the high precision of LDM process $(200 \mu \mathrm{m})$, we mentioned only one result for each object. Here, three $3 \mathrm{~d}$ printed experiments were also performed to test the printability with a standard deviation less than $2 \%$, demonstrating the good ability of printing setup.

We have added this statistical result in Fig6: "Three 3D printed experiments were performed for each object with a standard deviation less than $2 \%$ ".

5. Please add some equations or examples in the methods part to explain how the "Mean relative error" was calculated in Table 2 and Supplementary information 4 to make it easier to understand.

Thank you for this remark. We have added two equations in supplementary information 4.

6. In the "Conclusion" part, please change "confront" (line 21) to a more proper word.

We have changed "confront" by "compare". 
Reviewer \#4: The authors developed a novel prediction tool for the soft materials used in the LDM additive manufacturing process. This work is interesting and contributes to the scientific comunity of additive manufacturing. Several comments and questions are listed below awaiting to be addressed before the publication.

1. In the introduction, the authors can have more introduction about voxel 3D stimulation. Any relevant methods? Why authors use this method? Compared to other methods such as FEA, what is the advantage in the stimulation for the LDM process?

Thanks, it's a relevant question. Compared to FEA method, voxel-based approach was easier to use and allowed us to save time in order to obtain the simulation results, especially for complex and large $3 \mathrm{~d}$ object. Also, voxel-based approach can run with a lower computing capacity with the same precision as FEA.

A sentence of the introduction has been modified in the revised manuscript.

2. The experimental materials used in this experimental work are silicone elastomer. Is this material a Newtonian solution? Is this predicting tool only available for Newtonian fluids? What about non-Newtonian solutions such as shear-thinning materials or shear-thickening materials? When the processing parameters such as printing speed and printing pressure are changing, will it be influence the accuracy of this predicting tool since they will change the rheological behavior of materials.

In this paper, prediction tool was not performed to simulate silicone formulations to flow. So Newtonian, Shear-thinning or shear-thickening are not relevant here.

As mentioned in the introduction, the prediction tool simulates only the material at rest. Thereby, only static yield stress behavior was investigated to anticipate collapse of printed objects without taking into account the processing parameters.

"In the present study, a numerical simulation program was developed and validated as a preprinting decision tool for soft material 3D printing. The program defines material printability, through shear yield stress value, and compares it with the voxelized 3D object (called FingerMap) to be printed. It then predicts the non-printability of the object by displaying a map of potential collapse areas. This tool is intended to allow users to anticipate in silico the material printability according to each $3 D$ design, and not via the optimisation of printing parameters, thus reducing the number of tests required and accelerating the development of LDM technology using soft materials."

3. STL files are normally in the form of triangles representations. In this work, is each triangle represented by a voxel or each point represent a voxel?

Triangles in STL files are used to define only the surface of $3 \mathrm{~d}$ object which not enough to predict the collapse of printed objects. In this paper, voxel-approach was used to investigate the volume of printed objects where each point is represented by a voxel.

This information has been added to the revised manuscript (Page 7, line 4)

4. In section 4.1, the equation is suggested to move to the Materials and method section.

Equation has been moved in Material and Methods section.

5. In section 4.3 and 4.4 , the authors mentioned the control printing component using ObjetPro. D. However, there is no statement regarding the control in the materials and method sections. The statement regarding the control print is available in section 2.4: "Ear and aortic valve models have also been printed using an Objet Pro inkjet printer (Stratasys, IL) with VeroClear resin (Stratasys, IL) in order to have reference geometries for comparison." 
6. In section 4.4, line 32-33, "As can be seen, print heights of 0.7 and $0.5 \mathrm{~mm}$ were obtained for the LSR and LSR+PEG formulations, respectively" should be modified. The authors pointing out 0.7 and $0.5 \mathrm{~mm}$ should be the difference between the design height.

Yes, you are right. Our apologies for this mistake. We have changed the sentence by: "As can be seen, the difference of print heights of 0.7 and $0.5 \mathrm{~mm}$ were obtained for the LSR and LSR+PEG formulations, respectively". 


\section{Reviewer \#5:}

1. After finishing the literature review, please give a summary stating the major problems in this field now, and which problem will be solved in this study. Please reorganize the paragraphs in the section introduction. Make sure it is clear enough to be understand. Same for other sections. In the introduction section, the major problem of elastomer printing through LDM process was clearly described and related to others works:

"The complexity of using these materials through additive manufacturing lies in understanding their rheological properties [4] throughout their state transition, from liquid to solid. The best known soft material is silicone, which is present in all sectors of activity [5]".

As well as for the problem solved:

"In the present study, a numerical simulation program was developed and validated as a preprinting decision tool for soft material 3D printing. The program defines material printability, through shear yield stress value, and compares it with the voxelized 3D object (called FingerMap) to be printed. It then predicts the non-printability of the object by displaying a map of potential collapse areas. This tool is intended to allow users to anticipate in silico the material printability according to each $3 D$ design, and not via the optimisation of printing parameters, thus reducing the number of tests required and accelerating the development of LDM technology using soft materials".

Additional information and details were added to clarify the Introduction in the revised manuscript.

2. Three formulations of Silbione ${ }^{\circledR}$ LSR 4350 were used. The first one was the crude material, the second was the material mixed with PEG (Mw=400 $\mathrm{g} / \mathrm{mol}$ ) (SigmaAldrich, USA) and the third formulation was material mixed with BLUESIL ${ }^{\circledR}$ SP 3300 (Elkem Silicones, France), a nonhydrolysable silicone polyether copolymer. Suggest putting them in a table. Would be easier to know the differences.

We have added a table for the description of Silicone-based formulations as Supplementary Information 1. Numbering of the others have been adjusted.

3. In the present study, we approximated the normal yield stress [25] and the yield. Third person writing, please.

The sentence has been changed to:

"In the present study, the normal yield stress [25] and the yield stress tensor [26] were approximated by measuring only the static shear yield stress $\left(\tau_{y}^{s}\right)^{\prime \prime}$.

4. The other two designs were evaluation objects, namely an ear (Fig. 1.C) and an aortic valve (Fig. 1.D), both 3D models which were used to validate the result given by our predictive tool. Using two sentences.

We split this sentence in two:

"The other two designs were evaluation objects, namely an ear (Fig. 1.C) and an aortic valve (Fig.

1.D). These ones were used to validate the result given by our predictive tool".

5. Cartesian 3D printer (Tobeca ${ }^{\circledR}$, France) was used. This printer possesses a movement precision of $10 \mathrm{?m}$. All formulations were loaded in $10 \mathrm{cc}$ cartridges equipped with a standard piston and a $\mathbf{2 0 0} \mu \mathrm{m}$ frustoconical nozzle (Nordson EFD, USA). The dispensing flow rate was controlled by an Ultimus $V$ pneumatic equipment (Nordson EFD, USA). Two many names of the systems/testers. Suggest putting all of them in a table as supplemental materials.

We have adjusted the Supplementary Information 2 to meet your request. 
6. Fig. 6. 3D printing dimensional analysis after complete curing. A. 3D printed ears obtained using the three different formulations next to the control print. Numbers represent the height of the overhanging 3D structure. B. 3D printed aortic valves obtained using the three different formulations next to the control print. Numbers represent the height of the 3D structure Suggest using a table listing all the findings through the comparison, including the discussion in section 5 , which will give the reader a better understanding of the different methods.

As mention in section 5, a list of all findings through the comparison is already available in Supplementary Materials 4:

"In order to quantify the printing accuracy, strategic calliper-based measurements were performed on characteristic dimensions (supplementary information 4) and the mean relative deviation between the theoretical geometries, given by STL files, and the printed objects were extracted (Table 2)."

7. Section 6 Suggest listing all the major findings using bullets.

It has been done. 


\title{
FingerMap: A new approach to predict soft material 3D
}

\section{objects printability}

\author{
Alix Lopez ${ }^{\mathrm{a}}$, Christophe A. Marquette ${ }^{\mathrm{a}}$, Edwin-Joffrey Courtial ${ }^{\mathrm{a}}$ \\ a 3d.FAB, Univ Lyon, Université Lyon1, CNRS, INSA, CPE-Lyon, ICBMS, UMR 5246, \\ 43, Bd du 11 novembre 1918, 69622, Villeurbanne cedex, France
}

Keywords: 3D printing; Liquid Deposition Modelling; Silicone; Simulation; Yield Stress

Corresponding author:

Dr. Christophe Marquette: christophe.marquette@univ-lyon1.fr 